\title{
Chemical Abstracts cumple cien años
}

\author{
Por Luis-Javier Martínez
}

\begin{abstract}
Resumen: Un repaso histórico por los cien años de Chemical Abstracts Service (CAS) pone de relieve su significación como: (i) testigo del cambio en el liderazgo científico internacional; (ii) exponente clásico de la documentación científica; (iii) inventario general de las moléculas de nuestro mundo natural y artificial; (iv) estrecho aliado de la industria químico-farmacéutica; y (v) actor paradójicamente (des)colocado en la encrucijada del mundo digital. El futuro incierto de CAS también simboliza la incertidumbre reinante en materia de comunicación científica.
\end{abstract}

Palabras clave: Bases de datos, Química, Chemical Abstracts Service, American Chemical Society.

\section{Title: Chemical Abstracts celebrates one hundred years}

Abstract: A review of the first hundred years of Chemical Abstracts Service (CAS) reviews its significance and role as: (i) a witness to the changes in international scientific leadership; (ii) a classical example of scientific documentation; (iii) a general catalogue of chemical molecules in our natural and artificial world; (iv) a close ally of chemical and pharmacological industries; and (v) a paradoxically unsettled agent at a crossroads of the digital world. The uncertain future of CAS also symbolizes the uncertainty that reigns in scientific communication nowadays.

Keywords: Databases, Chemistry, Chemical Abstracts Service, American Chemical Society.

Martínez, Luis-Javier. “Chemical Abstracts cumple cien años”. En: El profesional de la información, 2007, septiembre-octubre, v. 16, n. 5, pp. 481-488.

DOI: $10.3145 /$ epi.2007.sep.11

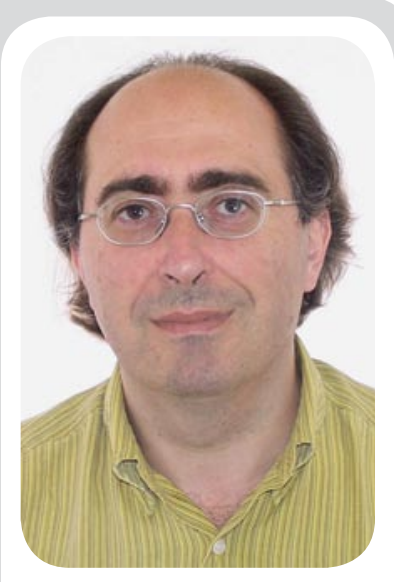

Luis Javier Martínez es licenciado en filosofía (lógica y teoría de la ciencia) por la Universidad de Salamanca. Ha sido subdirector y director de la Biblioteca de la Universidad de Cantabria. Actualmente es jefe de área de ciencia y tecnología en dicho organismo y editor del blog Observatorio de Martinej.

http://martinej.wordpress.com
LA BASE DE DATOS CHEMICAL ABSTRACTS SERVI$C E$ (CAS), uno de los grandes monumentos de la documentación y de la ciencia, ha cumplido cien años en 2007. En comparación con la velocidad y los cambios propios del momento actual, causa sorpresa y admiración una empresa colectiva y compleja de alcance internacional que ha sido capaz de desarrollarse con coherencia durante tanto tiempo. Parece un momento oportuno para realizar una mínima reflexión sobre lo que significan para la documentación y la ciencia estos cien años de Chemical Abstracts.

http://www.cas.org

\section{Testigo del cambio en el liderazgo científico internacional}

Si el francés Lavoisier y los británicos Priestley, Cavendish,
Dalton y Davy entre otros, habían contribuido decisivamente al surgimiento de la química moderna a partir del siglo XVIII, fue Alemania desde mediados del siglo XIX el país que dominó el panorama internacional de la química científica e industrial, como también sucedió en otras ramas del conocimiento. Los trabajos de Liebig, Kekulé, Wöhler, Kolbe y otros en química estructural y síntesis orgánica fueron decisivos para la profesionalización de la ciencia y para desencadenar una revolución industrial química. Grandes empresas actuales como Merck, BASF, Schering, Hoechst o Bayer se crearon en el siglo XIX. Asimismo en 1830 se empezó a publicar el que se conocería algo más tarde como Chemisches Zentralblatt, primer repertorio internacional de bibliografía química, y en el mismo siglo iniciaron su publicación los gigantescos tratados Gmelin handbuch der anor- ganischen chemie (desde 1819) y Beilstein handbuch der organisches chemie (a partir de 1881).

Alemania era pues claramente hegemónica en química cuando, en 1907, surgió en EUA Chemical Abstracts bajo los auspicios de la American Chemical Association $(A C S)$, de la que sigue siendo una división aunque económicamente independiente. Su misión era difundir resúmenes de toda la literatura mundial sobre química. Desde el primer número ya se pudo apreciar su intencionalidad de alcance internacional y su firme vocación de exhaustividad en cuanto a temas, tipos de documentos, idiomas y países de publicación de la bibliografía. Centrado siempre en la química, cubre hoy un extenso conjunto de áreas: bioquímica, química orgánica y aplicada, farmacología, petroquímica, polímeros, química inorgánica, física y analítica, materiales, temas biomédicos, etc. 
Algunos autores, como Baker (1980, p. 193) señalan que $C A S$ surgió de la insatisfacción de los químicos norteamericanos con la cobertura que los repertorios europeos hacían de la literatura de su país. Sea o no éste el caso, lo cierto es que pronto mostró una clara ambición de liderazgo del sector; el deseo, en suma, de ser la clave de la ciencia y la ingeniería química en el mundo. Su nacimiento vendría a señalar además el despegue de la química norteamericana, un crecimiento que también fue paralelo en otras ciencias.

Baker (1980, p. 197) explica que en su primer año casi la mitad de los resúmenes publicados eran de artículos alemanes. En 1913 constituían un tercio, en 1929 una cuarta parte y en 1979 sólo un 7,2\% (incluyendo las dos Alemanias de esa época). A mediados del siglo XX se convirtió en la fuente bibliográfica internacional por antonomasia en el sector químico a la par que dejaban de publicarse los repertorios europeos British Abstracts de la Royal Society of Chemistry, que desapareció en 1953, y Chemisches Zentralblatt, que lo hizo en 1969. En ambos casos, sus sociedades editoras aceptaron ocuparse de analizar y resumir para $C A S$ la documentación publicada en sus países.

La persecución nazi de los judíos y la segunda guerra mundial, con el éxodo masivo de científicos desde Europa a EUA, supusieron la pérdida definitiva de la hegemonía científica por parte de Alemania y, en buena medida, de todo el continente. $\mathrm{La}$ evolución de $C A S$ es expresión de ese cambio en el liderazgo internacional de la ciencia y la tecnología, y también, por ende, en la titularidad de la supremacía política, militar y económica en el mundo.

\section{Exponente clásico de la documentación científica}

$C A S$ se empezó a publicar pocos años después de la iniciativa de
Otlet y Lafontaine sobre el Instituto Internacional de Bibliografía (1895), posteriormente denominado Federación Internacional de Documentación (FID). En 1898 había comenzado a editarse también en el Reino Unido Science Abstracts, ahora llamado Inspec. En consecuencia se puede decir que $C A S$ surge cuando la documentación aparece como un nuevo paso en la comunicación científica, y se convierte en uno de los exponentes más acabados y clásicos de la documentación, un depurado intento de procurar el control bibliográfico de la literatura científica.

Se aprecia en su historia un crecimiento continuo en referencias, sustancias, fuentes analizadas, personal, volumen económico y capacidades tecnológicas. En su primer año publicó 12.000 resúmenes en dos volúmenes impresos, mientras que ahora se incorporan anualmente alrededor de un millón (2.700 al día). Es la mayor base de datos científica del mundo. Sus dos archivos fundamentales o sub-bases de datos, interconectados, son $C A$ plus, con referencias y resúmenes de 27 millones de documentos, y Registry, con información de unos 31 millones de sustancias. Otros archivos del sistema $C A S$ son el de reacciones químicas, con datos de 12 millones de reacciones, el de regulaciones administrativas sobre compuestos químicos y el de catálogos comerciales de productos en venta.

La sede central ha estado desde sus primeros años en Columbus, Ohio, en el campus de la Ohio State University y desde 1965 cuenta con un edificio propio. Durante mucho tiempo, su publicación dependió del trabajo de cientos de voluntarios que leían y analizaban los textos primarios y redactaban los resúmenes. Cualesquiera que fueran las circunstancias (guerras, dificultades económicas, escasez de papel, etc.) CAS mantuvo una perseverante continuidad. A partir de mediados del siglo XX se aceleraron los cambios y en los años 60 comenzó la automatización de las complicadas y costosas tareas de análisis, mantenimiento y edición de la información. Simultáneamente se creó Registry, el archivo de sustancias, lo que constituye uno de los principales hitos en esta historia.

En cuanto a técnicas de resumen, ha apostado por aquellos de tipo informativo, frente a los analíticos o indicativos, y ha establecido pautas sobre cómo tiene que ser el abstract de un texto científico (Baker, 1980, p. 194). También se puede destacar que en materia de clasificación e indización de la bibliografía ha llevado a cabo una ejemplar labor (Zaye, 1985).

$C A S$ ha demostrado cómo se puede pasar de ser un gigante impreso, difícil de manejar en su producción y en su consulta, a una estructura cliente-servidor sobre internet (SciFinder). Saltó a la distribución on line a finales de los 70 , y al cd-rom a finales de los 80 . Ha creado su propio distribuidor de bases de datos (STN, Scientific and Technical Network), juntamente con FIZ Karlsruhe (Fachinformation Zentrum) y el Japan Information Center for Science and Technology (Jicst). En los años 90 lanzó SciFinder, un sistema pionero en la búsqueda amigable a texto libre de documentación científica, que incorpora herramientas de análisis de la información mediante minería de datos.

Durante la época de los complicados lenguajes y técnicas de recuperación automatizada $C A S$ reforzó, en un sector ávido de información, el papel mediador del profesional de la documentación, ante la dificultad de que los científicos buscaran por sí mismos con costes y resultados eficientes. Con SciFinder, en cambio, devuelve a los usuarios finales el poder de manejar directamente los datos, desplazando en buena me- 


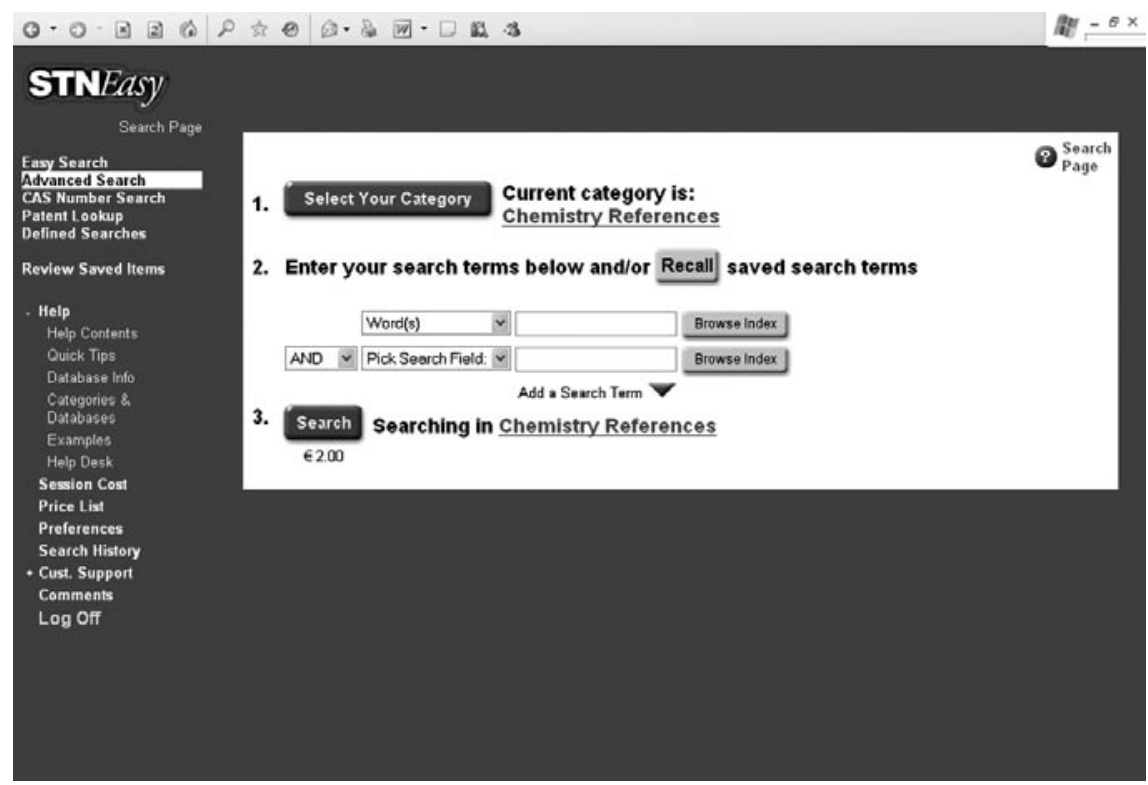

Figura 1. Una pantalla de búsqueda de STN Easy

dida a los mediadores, aunque éstos puedan seguir ofreciendo búsquedas muy especializadas mediante STN Express o STN on the Web. Lo que no ha hecho $C A S$, que es también un intermediario propiamente dicho, es abrirse a la web, volverse más transparente y accesible.
La ciencia y la ingeniería químicas se han mostrado como grandes consumidoras de información y de documentación, de registros del conocimiento (Fugmann, 1985). $C A S$ no es ajeno al hecho de que en buena medida la técnica de la documentación se ha desarrollado con la

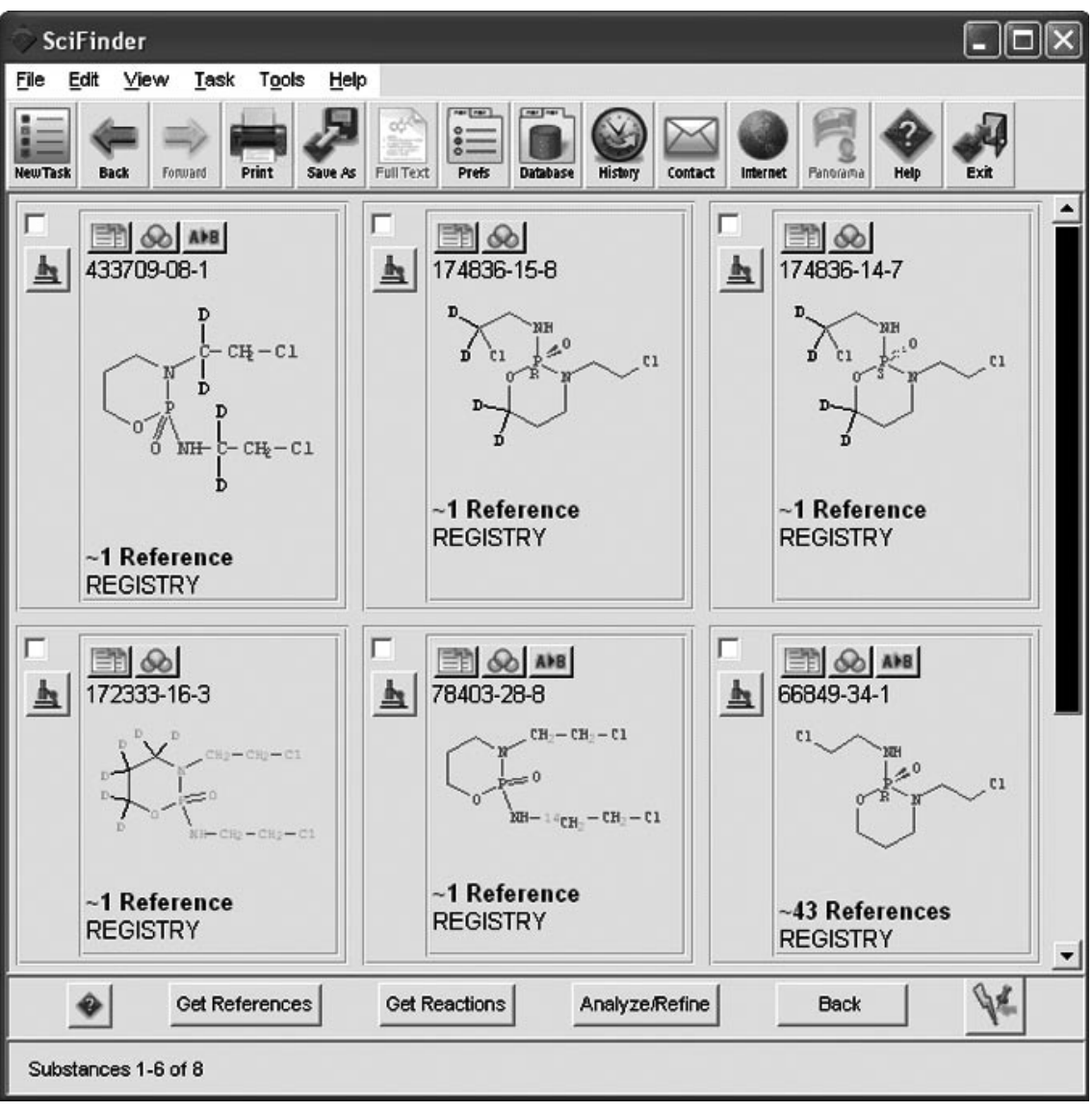

Figura 2. Búsqueda de sustancias en SciFinder química y que muchos documentalistas han sido químicos (Bradford y Garfield, por ejemplo). CAS es pues un ejemplo de la aportación de la documentación a la química y de la química a la documentación, un paradigma de las técnicas documentales que han respaldado el desarrollo de la ciencia y la tecnología en el pasado siglo.

\section{"La documentación se ha desarrollado con la química, y la química con la documentación"}

\section{Inventario universal de las moléculas}

La ciencia y la ingeniería químicas, y por ende, su literatura, tratan de sustancias, compuestos, moléculas. Es decir, tratan de su análisis e identificación, de la determinación de sus propiedades, de su preparación o síntesis, de su intervención o utilización en reacciones y procesos, y de su aprovechamiento práctico con múltiples fines. Almacenar y recuperar eficazmente información factual sobre las sustancias, sus rasgos fundamentales y/o la literatura donde se describen, ha sido siempre muy útil para el avance de la química (Weisgerber, 1997, p. 349).

En particular es muy necesaria la información sobre la estructura de las moléculas, los átomos que forman parte de ellas, su posición, enlaces y organización en el espacio, pues es lo que les confiere su identidad y propiedades. Poder representar, conservar, organizar y recuperar gráficamente o de algún modo las estructuras químicas, que son tridimensionales, reviste gran interés. Además identificar unívocamente las sustancias mediante sistemas de notación o códigos precisos es crucial para indizar, recuperar y gestionar la información sobre 


\begin{tabular}{|c|l|}
\hline \multicolumn{2}{|c|}{ Algunos hitos en la historia de Chemical Abstracts } \\
\hline $\mathbf{1 9 0 7}$ & William A. Noyes es el primer editor responsable de CAS \\
\hline $\mathbf{1 9 0 9}$ & CAS se instala en la Ohio State University, Columbus \\
\hline $\mathbf{1 9 2 9}$ & $\begin{array}{l}\text { En la redacción de los abstracts empiezan a participar colaboradores retribuidos, no } \\
\text { voluntarios }\end{array}$ \\
\hline $\mathbf{1 9 5 5}$ & $\begin{array}{l}\text { Ante la crisis presupuestaria de ACS, se decide que CAS tenga autonomía } \\
\text { económica, que se autofinancie como una división empresarial independiente }\end{array}$ \\
\hline $\mathbf{1 9 6 5}$ & $\begin{array}{l}\text { Se crea Registry, el archivo de sustancias, con información de estructuras } \\
\text { moleculares y códigos unívocos (CAS Registry Numbers) de las moléculas }\end{array}$ \\
\hline $\mathbf{1 9 6 5}$ & Edificio propio junto al campus de la Ohio State University, Columbus \\
\hline $\mathbf{A n ̃ o s} \mathbf{6 0}$ & Se informatizan los procesos de análisis documental y producción editorial. \\
\hline $\mathbf{A n ̃ o s ~ 7 0}$ & Desaparecen prácticamente los voluntarios entre los redactores de los abstracts \\
\hline $\mathbf{1 9 7 6}$ & $\begin{array}{l}\text { Chemical Abstracts empieza a ser consultado en línea, en sus primeras versiones } \\
\text { Ilamado CA Condensates (sin el texto de los abstracts) }\end{array}$ \\
\hline $\mathbf{1 9 8 3}$ & CAS Registry empieza a ser consultado en línea por los usuarios \\
\hline $\mathbf{1 9 8 4}$ & $\begin{array}{l}\text { Entra en servicio STN International, distribuidor en línea de numerosas bases de } \\
\text { datos científico-técnicas, creado por CAS con el FIZ Karlsruhe }\end{array}$ \\
\hline $\mathbf{1 9 8 8}$ & Se introduce el software STN Express para la búsqueda de información en STN \\
\hline $\mathbf{1 9 9 3}$ & $\begin{array}{l}\text { Se empiezan a integrar en Registry biosecuencias de moléculas de interés biológico } \\
\text { (proteínas, ácidos nucleicos, etc.) }\end{array}$ \\
\hline $\mathbf{1 9 9 5}$ & $\begin{array}{l}\text { Nace SciFinder, un sistema cliente-servidor de búsqueda avanzada de información } \\
\text { química, manejable por los científicos sin mediación }\end{array}$ \\
\hline $\mathbf{1 9 9 6}$ & Nace STN Easy, un sistema simplificado de búsqueda en 40 bases de datos de STN \\
\hline $\mathbf{1 9 9 7}$ & $\begin{array}{l}\text { Empieza a funcionar Chemport, un servicio electrónico de suministro de documentos } \\
\text { primarios también de CAS }\end{array}$ \\
\hline $\mathbf{2 0 0 5}$ & $\begin{array}{l}\text { Aparece Science IP, servicio de consultoría en búsquedas de información } \\
\text { perspectivas de la información recuperada en las búsquedas }\end{array}$ \\
\hline deada del $\mathbf{2 0 0 0}$ & \\
\hline &
\end{tabular}

las mismas, con fines científicos o comerciales. Todo esto es peculiar de la química.

Weisgerber (1997) realiza un detallado recorrido por los intentos sucesivos de normalizar y automatizar la notación química para manejar eficazmente la información estructural y demás datos de los compuestos. Es una larga historia que arranca a finales del siglo XIX y cuyo éxito fundamental y más ampliamente aceptado es el sistema CAS Registry. Buntrock (2001) también aborda este tema.

$C A S$ empezó a desarrollar Registry en 1965. Es un inventario de sustancias en el que constan los diversos nombres (comunes, sistemáticos, etc.), las propiedades, la fórmula y la estructura molecular de cada compuesto, permitiendo recuperar todas las referencias relacionadas con el mismo en el fichero bibliográfico, donde aparecen las sustancias como términos de indización. Actualmente contiene más de 30 millones de sustancias que en algún momento han sido descritas o citadas en la literatura química: compuestos orgánicos e inorgánicos, polímeros, aleaciones, minerales, compuestos de coordinación, etc. Por supuesto, incluye tanto moléculas existentes en la naturaleza como las artificiales, creadas sintéticamente por el hombre.

Frente a la complejidad y ambigüedad de la nomenclatura química,
Registry ha establecido una identificación unívoca de los compuestos, distinguiendo estereoisómeros y variantes iónicas, asignándoles el CAS Registry number, un código numérico consecutivo que es usado casi universalmente como su denominación estándar. Tiene hasta nueve dígitos, separados por guiones en tres grupos, el penúltimo de dos dígitos y el último un carácter de control. Por ejemplo, 50-78-2 es el $C A S R N$ de la aspirina (el compuesto 5.078).

Mucho antes de que se contara con las capacidades gráficas de la informática reciente, desde 1965 mundo, de las naturales y de las artificiales" 
CAS Registry consiguió asignar a cada molécula una representación de su estructura tridimensional mediante la técnica de las "tablas de conexión", que describen textualmente los átomos presentes, sus enlaces y sus posiciones. De esta manera no sólo se archivaba la estructura molecular de la sustancia, sino que esta estructura, o una parte de ella, una subestructura, se convertía en recuperable, en término de búsqueda en la base de datos. La consulta por subestructura abre paso a la selección de compuestos por propiedades funcionales dependientes de agrupaciones definidas de átomos.

En 1993 se incorporaron a la consulta en Registry secuencias de polímeros biológicos, proteínas y polinucleótidos, para las que se desarrolló un sistema de representación especial. En la actualidad cuenta con más de 50 millones de biosecuencias registradas.

El catálogo de los textos científicos (base de datos bibliográfica) se complementa y compenetra de manera potente con el catálogo de las moléculas (base de datos factual). Ningún otro inventario o sistema de identificación de sustancias es tan grande y tan generalmente aceptado. Podría decirse que Registry es el catálogo de referencia de las moléculas del mundo: la fuente autorizada de identificación y descripción de la diversidad química existente. Desde este punto de vista, CAS ha sido vital en el desarrollo del sector químico-farmacéutico.

\section{Aliado estrecho del desarrollo industrial}

Hasta la aparición de la tecnología nuclear y la biotecnología, las industrias químico-farmacéuticas han sido las que de manera más radical han transformado la naturaleza, y no precisamente sin consecuencias, como bien sabemos. A partir de sustancias naturales, preparan, a veces a gran escala, nuevos compuestos con una utilidad determinada (terapéutica, agrícola, alimentaria, energética, textil, higiénica, etc.). El valor añadido reside en gran parte en el conocimiento sobre cómo realizar transformaciones y procesos eficientes, más que en las materias primas de partida. Desde los procesos Leblanc, Solvay o Haber, estamos ante un sector empresarial intensamente basado en el conocimiento, en la innovación, y por tanto dependiente de sus registros y documentos, de la información científica y tecnológica.

$C A S$ ha sido en origen, incluso en su sede, una iniciativa cercana al mundo académico. Pero se ha orientado con decisión hacia las empresas desde que en el primer momento, con su pretensión de globalidad, quiso abarcar la literatura en química industrial y las patentes. $\mathrm{Su}$ posición desde 1955 como empresa independiente bajo la tutela de la $A C S$ tal vez haya reforzado esta vocación. El caso es que a lo largo de toda su historia se ha desenvuelto como una herramienta para la industria químico-farmacéutica, con gran éxito comercial y fuerte

EI sistema CAS: archivos componentes

\begin{tabular}{|l|l|}
\hline Registry & Sustancias químicas y biosecuencias \\
\hline CAplus & Referencias de literatura científica y patentes \\
\hline CASReact & Reacciones químicas de todo tipo \\
\hline ChemCats & Catálogos comerciales de productos químicos \\
\hline ChemList & Regulaciones administrativas sobre sustancias \\
\hline Marpat & Estructuras de Markush de genéricos en patentes \\
\hline
\end{tabular}

implantación en las empresas privadas, cosa que ha impregnado su cultura de trabajo. Marris (2005) recoge la crítica de algún químico ilustre que piensa incluso que $A C S$ ha adquirido una cultura de negocio con fines lucrativos.

\section{"Desde su origen CAS tuvo una firme vocación de globalidad, exhaustividad y liderazgo"}

Uno de sus principales rasgos es su cobertura de la documentación de patentes, lo que siempre le ha distinguido de otras bases de datos. Las patentes, cruciales en la industria y portadoras de conocimientos susceptibles de aplicación y explotación, han sido siempre tratadas por $C A S$ en profundidad, con atención y rigor, lo que lo ha convertido en una de las fuentes comerciales de información básicas en materia de propiedad industrial (la otra es Derwent).

Incluye ahora referencias de más de 4 millones de documentos de patentes emitidos por unas 50 oficinas de propiedad industrial de todo el mundo. STN, el distribuidor de bases de datos de $C A S$, ofrece otros muchos ficheros y productos con información de patentes (Derwent, Inpadoc, etc.), lo que lo convierte en un instrumento ampliamente usado por quienes necesitan hacer estudios sobre el estado de la técnica, la patentabilidad de invenciones, vigilancia tecnológica, infracción de patentes, estatus legal de familias de patentes, etc. Recientemente, ha puesto en marcha Science IP, un servicio de búsquedas y alertas de información en materia de propiedad industrial, para quienes no deseen hacerlas por sí mismos.

Con el software STN AnaVist, Chemical Abstracts facilita a las 
empresas y departamentos de I+D el análisis y visualización de la información extraída de sus bases de datos, para estudiar tendencias de la investigación y hacer vigilancia tecnológica e inteligencia competitiva.

$C A S$ ha sido, pues, un aliado cercano para las empresas farmacéuticas y químicas del siglo XX. Pero se abren las primeras incertidumbres: falta saber qué función tendrá en la recién inaugurada época de la biotecnología, que opera sobre uno de los nuevos territorios industrialmente explotables abiertos en las fronteras del conocimiento. $\mathrm{Se}$ desconoce el papel que jugará en la parcial reconversión de la ingeniería química en ingeniería ambiental, después de los terribles impactos sobre el planeta que ha causado la primera en doscientos años. Y falta saber también qué lugar ocupará en el control informativo de la propiedad industrial, cuando todas las oficinas de patentes colocan sus bases de datos a texto completo, en acceso abierto, en internet.

\section{(Des)colocado en la encrucijada de la época digital}

Desde la aparición de la web y la consecuente explosión de internet y la información digital, aparecen nuevas tendencias y tecnologías en la comunicación del conocimiento, generalmente mucho más prolíficas, más abiertas a la replicación de la información. El ambiente ha cambiado y además lo hace todos los días. ¿Cómo lo está abordando CAS?

$\mathrm{Su}$ respuesta fundamental a las nuevas circunstancias ha sido SciFinder, un sistema de documentación química, pionero en muchos aspectos. Fue lanzado hace diez años, primero para el mundo de la empresa y después para entornos académicos (SciFinder Scholar). Siempre ha sido un recurso caro, que difícilmente ha podido ser suscrito de manera extendida y en tarifa plana en las universidades. Flaxbart (2007) critica además el suspicaz control que ha ejercido $C A S$ sobre su uso en las bibliotecas universitarias (número de accesos, usuarios externos, etc.).

En un mundo de tecnologías y fuentes de información populares, SciFinder se ha mantenido como un recurso de élite. Pero centrarse demasiado en el mercado industrial le quita a $C A S$ aliados en el mundo académico (profesores, bibliotecarios, etc.) y le resta base de futuro entre los químicos en formación. Y las empresas por sí solas dejan de ser clientes fieles en cuanto encuentran opciones mejores. Probablemente no ha habido un buen equilibrio en la orientación a los dos sectores del mercado, o quizá $C A S$ ha hecho bien las cuentas y acierta, pero no sin riesgos.

Otra forma de responder a las nuevas tendencias en información científica ha sido oponerse a inicia- tivas que buscan una distribución más abierta del conocimiento. En el sector de la química hay menos proyectos de open access que en otras disciplinas. Existen pocos archivos temáticos relevantes de eprints $\mathrm{y}$ pocas revistas electrónicas abiertas influyentes. Puede que ello sea parte de la cultura epistémica de la química. Pero sí hay buscadores y fuentes de datos factuales bastante abiertos, como Chemfinder, NIST Chemistry Webook, PubChem, ChemExper, eMolecules, ChemWeb, etc. Y ha surgido un sistema abierto de codificación de sustancias, el International Chemical Identifier (InChI), que no es propiedad de una empresa privada que cobre por dejarlo usar, como el CAS RN, sino que está elaborado bajo los auspicios de la $I u$ pac (International Union of Pure \& Applied Chemistry) para su uso en contextos open access.

Chemical Abstracts o la ACS han atacado varios proyectos que

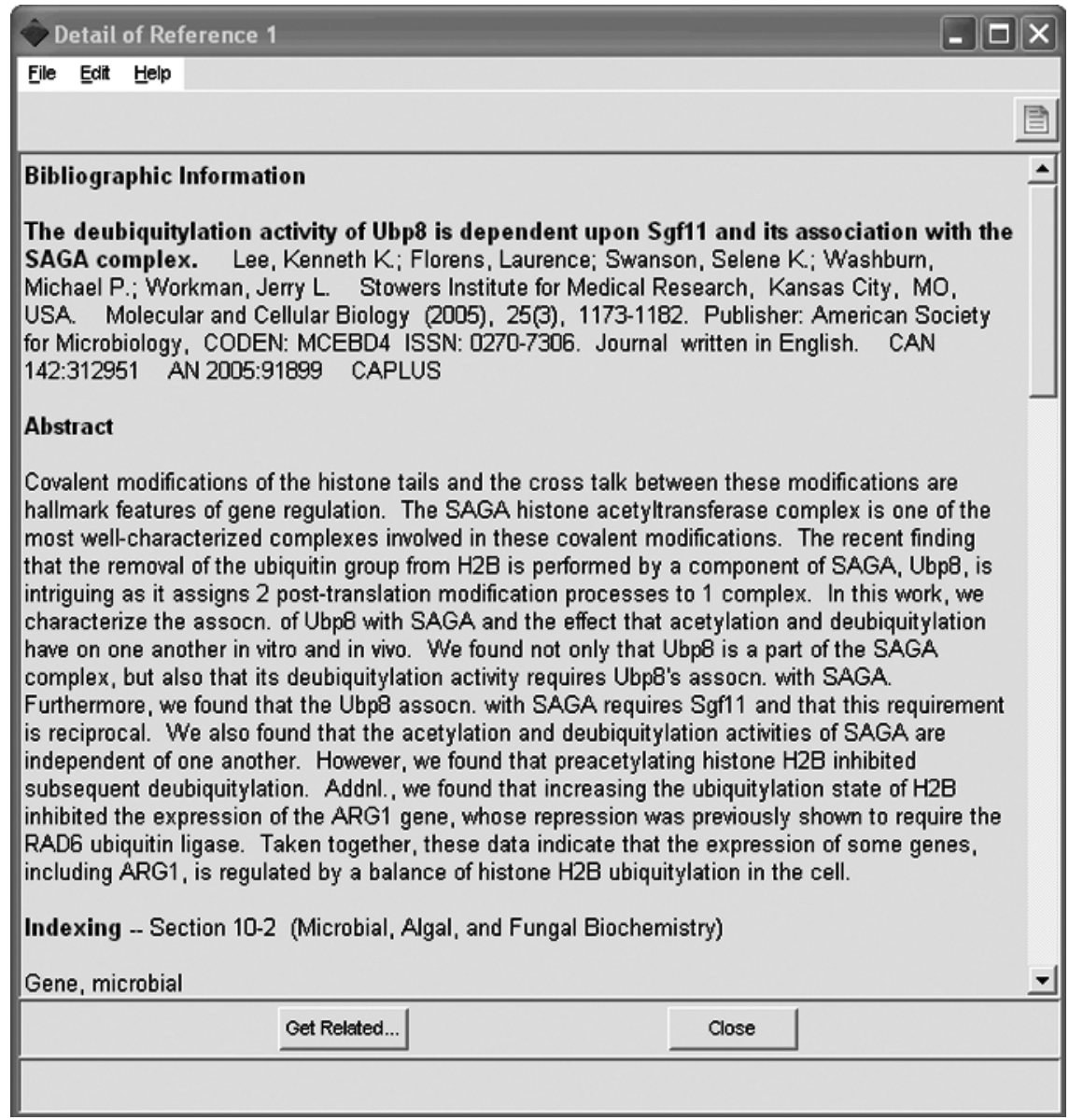

Figura 3. Una referencia bibliográfica en SciFinder 
afectan a su mercado, lo que no ha favorecido su imagen pública, al hacerles aparecer como resistentes partidarios del "antiguo régimen". Por ejemplo, en 2004 demandaron a Google por apropiación de marca comercial a causa del uso que el buscador hace del adjetivo Scholar, que $C A S$ vincula como exclusivo de su servicio SciFinder Scholar. En 2005 la $A C S$ emprendió una campaña contra la base de datos $P u b$ Chem, de los National Institutes of Health (NIH), que ofrece en acceso libre información sobre moléculas de interés biológico, argumentando que PubChem invade su negocio duplicando con dinero público un servicio que ya está ofreciendo $C A S$ en el sector privado, violando así la libertad de empresa. El asunto ha despertado controversias y críticas dentro de la propia $A C S$, explica Marris (2005).

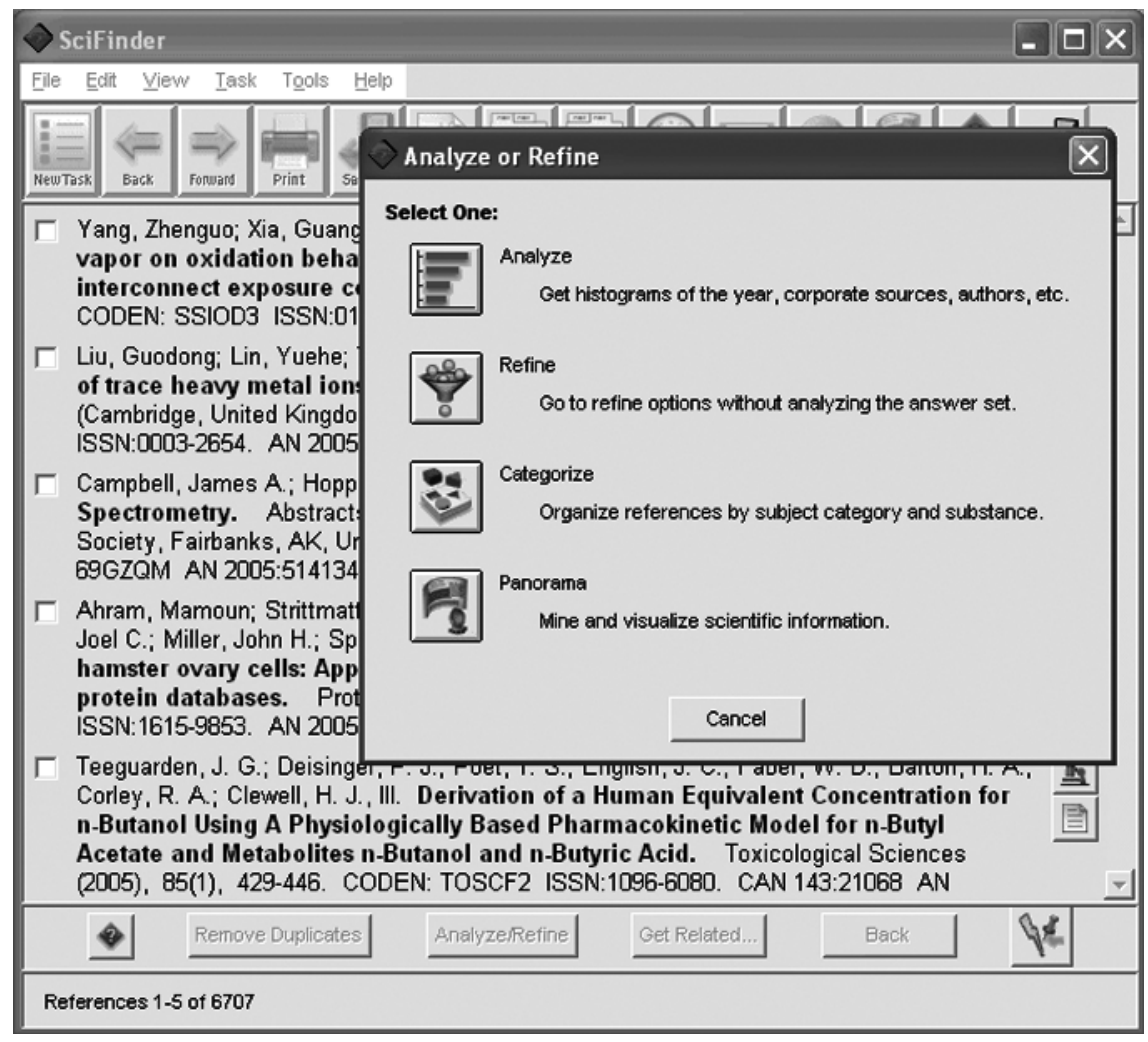

Figura 4. Herramientas de análisis de información en SciFinder

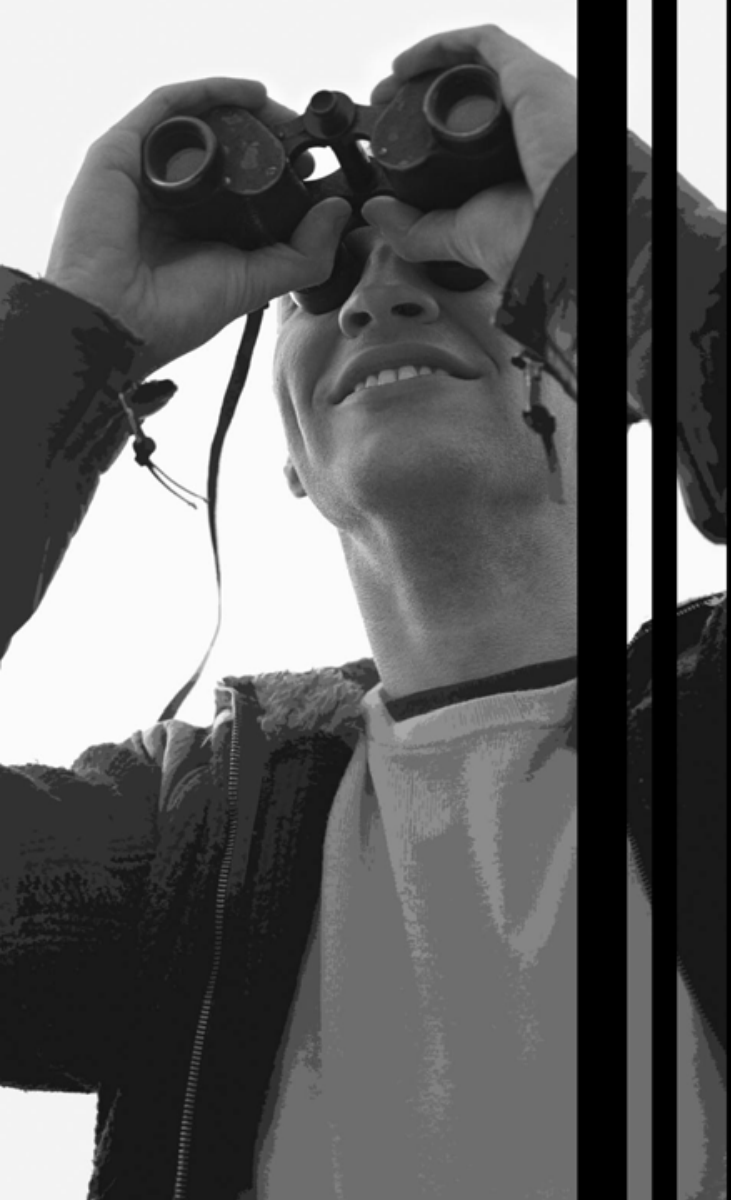

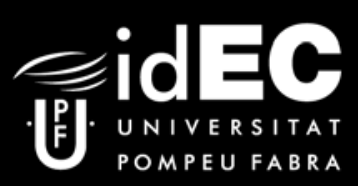

MÁSTER EN DOCUMENTACIÓN DIGITAL

Máster y Programas de posgrado online

Arquitectura de la información en sitios web. - Organización de la información y sistemas de gestión de bases de datos. - Buscadores y posicionamiento web. - Documentación audiovisual. - Gestión de proyectos en centros de información. - Archivística digital. - Gestión de contenidos y gestión del conocimiento. - Bibliotecas digitales. - Usabilidad.

Dirección: Cristòfol Rovira y Lluís Codina

Calendario: oct 07 - jun 08

www.documentaciondigital.org

\section{MÁSTER EN BUSCADORES}

POSICIONAMIENTO, MARKETING ONLINE Y VIGILANCIA TECNOLÓGICA Máster y Programas de posgrado online

El objetivo general del curso es proporcionar una sólida formación sobre todos los aspectos relacionados con los buscadores de Internet en sus diferentes facetas: herramientas de recuperación de información, medios privilegiados para el marketing online, instrumentos para implementar programas de vigilancia tecnológica e inteligencia competitiva.

Dirección: Cristòfol Rovira y Lluís Codina

Calendario: nov 07 - jun 08

www.masterenbuscadores.com

\section{Colaboran:}

Observatorio de Comunicación Científica · UPF

Instituto Universitario de Lingüística Aplicada · UPF

DOC 6 Consultores de Recursos de Información

Infórmate

www.idec.upf.edu | info@idec.upf.edu | Tel. 935421850 | Balmes 132, Barcelona 
Como sucede a todos los editores secundarios, que ofrecen servicios de gran calidad y coste (y a otros mediadores de la comunicación científica), su posición está amenazada en el nuevo escenario de internet. Los editores secundarios son potentes agregadores de valor, pero a un elevado coste, y sin la base segura de la propiedad intelectual sobre los contenidos fuente: los documentos no son suyos, sino de los editores primarios, y las moléculas tampoco son de $C A S$, ni las naturales, ni las que se inventan y patentan. Cualquiera puede crear alternativas, y por supuesto Google también. Puede consultarse al respecto el reciente estudio de Levine-Clark (2007) que compara Google Scholar y SciFinder Scholar. Es bien sabido que en ocasiones el producto de menor calidad pero más barato y sencillo derrota al de mayor calidad pero más caro y complejo.

CAS responde a este escenario tal vez con más rigidez que habilidad adaptativa. Son muy esclarecedoras sobre estos temas las reflexiones de Flaxbart (2007). CAS tiene detrás una historia larga, sólida y coherente de innovación, crecimiento y servicio; ciertamente hay demasiada calidad y contenido detrás como para que se lo lleve el viento. Pero quizá los últimos y tempestuosos aires de cambio en el entorno de las TIC (disruptive technologies) están siendo demasiado violentos y lo descolocan, lo sitúan en una encrucijada, ante un futuro con interrogantes.

\section{Conclusiones}

Pese a todos los claroscuros, conflictos e incertidumbres, debe reco-

\section{"CAS refleja los conflictos de valores que tienen lugar en} el seno de la tecnociencia actual"

nocerse en primer lugar que $C A S$ es una obra intelectual digna de admiración, uno de los principales motores de la técnica y la tecnología documental y un hito en la historia reciente de la ciencia y de la industria. Pero por otra parte, como también dice Flaxbart (2007), muchos profesionales de la documentación querrían utilizar un sistema de información especializado y de calidad como $C A S$, pero necesitarían un producto que fuera algo más barato, más abierto y más flexible para canalizar a sus usuarios finales.

En todo caso, su incierto futuro también simboliza o significa algo: la incertidumbre general reinante en el campo de la comunicación científica, que afecta a todos los que intervienen en ella. Y que se extiende a todos los ámbitos de la vida en la época digital.

Con su doble alma de asociación científica y de empresa comercial, a caballo entre el mundo de la información reservada, potencialmente rentable, y el de la información pública socialmente compartida, la situación de $C A S$ es un ejemplo asimismo de los conflictos de valores que tienen lugar en el seno de la tecnociencia actual entre fines económicos, cognitivos y sociales.

\section{Bibliografía}

Baker, Dale B.; Horiszny, Jean W.; Metanomski, Wladyslaw V. "History of abstracting at Chemical Abstracts Service". En: Journal of chemi- cal information and computer sciences, 1980, v. 20, n. 4, pp. 193-201.

Buntrock, Robert E. "Chemical registries-in the fourth decade of service". En: Journal of chemical information and computer sciences, 2001, March, v. 41, n. 2, pp. 259-263.

CAS $100^{\text {th }}$ anniversary celebration. Consultado en: 15-06-07.

http://www.cas.org/aboutcas/cas100/index.html

Flaxbart, David. "The Chemical Abstracts centennial: whither CAS?" En: Issues in science \& technology librarianship, 2007, n. 49. Consultado en: 15-06-07.

http://www.istl.org/07-winter/viewpoints.html

Fugmann, Robert. "Peculiarities of chemical information from a theoretical viewpoint". En: Journal of chemical information and computer sciences, 1985, August, v. 25, n. 3, pp. $174-$ 180.

Levine-Clark, Michael; Kraus, Joseph. "Finding chemistry information using Google Scholar: a comparison with Chemical Abstracts Service". En: Science \& technology libraries, 2007, v. 27 , n. 4 (prepublication, accepted for publication). Consultado en: 15-06-07.

http://eprints.rclis.org/archive/00008633/

Marris, Emma. "American Chemical Society: chemical reaction". En: Nature, 2005, Oct., v. 437, n. 7.060, pp. 807-809.

Powell, Evelyn Constance. "A history of Chemical Abstracts Service, 1907-1998”. En: Science \& technology libraries, 2000 , v. 18, n. 4, pp. 93-110.

Weisgerber, David W. "Chemical Abstracts Service Chemical Registry System: history, scope, and impacts". En: Journal of the American Society for Information Science, 1997, v. 48, n. 4, pp. 349-360.

Zaye, D. F.; Metanomski, W. V.; Beach, A. J. "A history of general subject indexing at Chemical Abstracts Service". En: Journal of chemical information and computer sciences, 1985, November, v. 25, n. 4, pp. 392-399.

Luis Javier Martínez, Biblioteca de la Universidad de Cantabria. luisjaviermartinez@ono.com

\section{Reducción del embargo online de EPI}

EPI ha bajando ya a 1 año (6 números) el tiempo de carencia o de embargo (time embargo, time wall) de acceso de pago a la versión online de la revista. En consecuencia se han liberado los contenidos del periodo enero 2000-julio 2006, que están en acceso abierto en la web de la propia revista:

http://www.elprofesionaldelainformacion.com

El periodo 1992-1999 está disponible en html y el 2000-2006 en pdf. 
1) Blogs y otros productos informativos en internet.

2) Organización de cursos.

a) Propios abiertos al público en general.

b) Para empresas, como formación interna o mediante la externalización de su propuesta formativa (co-organización).

c) Para colectivos que necesiten formación específica.

3) Organización de jornadas y otros eventos profesionales.

4) Selección de personal.

\section{www.informa.cat}

